\title{
Ses \\ Transport detection of quantum Hall fluctuations in graphene
}

\author{
Simon Branchaud, ${ }^{1,2}$ Alicia Kam, ${ }^{1}$ Piotr Zawadzki, ${ }^{1}$ François M. Peeters, ${ }^{3}$ and Andrew S. Sachrajda ${ }^{1}$ \\ ${ }_{1}^{1}$ Institute for Microstructural Sciences, National Research Council of Canada, Ottawa, Ontario, Canada K1A OR6 \\ ${ }^{2}$ Département de Physique, Université de Sherbrooke, Sherbrooke, Quebec, Canada J1K 2R1 \\ ${ }^{3}$ Department of Physics, University of Antwerp, Groenenborgerlaan 171, B-2020 Antwerp, Belgium \\ (Received 14 October 2009; revised manuscript received 4 February 2010; published 9 March 2010)
}

\begin{abstract}
Low-temperature magnetoconductance measurements were made in the vicinity of the charge neutrality point (CNP). Two origins for the fluctuations were identified close to the CNP. At very low magnetic fields there exist only mesoscopic magnetoconductance quantum interference features which develop rapidly as a function of density. At slightly higher fields $(>0.5 \mathrm{~T})$, close to the CNP, additional fluctuations track the quantum Hall $(\mathrm{QH})$ sequence expected for monolayer graphene. These additional features are attributed to effects of locally charging individual QH localized states. These effects reveal a precursor to the quantum Hall effect since, unlike previous transport observations of $\mathrm{QH}$ dot charging effects, they occur in the absence of quantum Hall plateaus or Shubnikov-de Haas oscillations. From our transport data we are able to extract parameters that characterize the inhomogeneities in our device.
\end{abstract}

DOI: $10.1103 /$ PhysRevB.81.121406

PACS number(s): 73.43.Qt, 73.63.-b, 73.22.-f

Since the first experimental realization of mechanically exfoliated graphene ${ }^{1}$ there has been growing interest in its material properties close to the charge neutrality point (CNP) ${ }^{2-4} \mathrm{~A}$ finite resistance at the CNP provided strong evidence of the existence of electron and hole puddles, which was confirmed directly by scanning probe techniques. ${ }^{5}$

One of many remarkable properties of graphene in comparison with other two-dimensional electron gases (2DEGs) is that in spite of its dramatically lower mobility, mesoscopic conductance fluctuations persist to surprisingly high temperatures $^{6-8}$ as predicted. ${ }^{9}$ On the other hand, effects which require time-reversal symmetry in addition to phase coherence, such as weak localization, are suppressed in the absence of mechanisms enabling intervalley scattering. ${ }^{10-12}$ Analyzing low-field magnetotransport measurements has become a critical tool to extract important scattering parameters. To make an interpretation straightforward measurements are typically restricted to magnetic fields lower than those at which quantum Hall effect (QHE) features are visible. Graphene samples made via mechanically exfoliated techniques, however, tend to involve voltage contacts placed together at distances roughly equivalent to inhomogeneity length scales thus allowing the conductance to be sensitive to mesoscopic phenomena. In addition the spread in densities is significant in graphene. This raises the question as to what low-field measurements of magnetoconductance fluctuations in graphene are actually probing. In this Rapid Communication, we find that the charging of individual quantum hall $(\mathrm{QH})$ localized states can dominate the magnetoconductance in transport experiments down to magnetic fields four times lower than any other QHE-related features such as QHE plateaux or Shubnikov-de Haas $(\mathrm{SdH})$ oscillations.

The graphene flake was mechanically exfoliated over an $n$-doped $\mathrm{Si}$ substrate covered with $300 \mathrm{~nm}$ of $\mathrm{SiO}_{2}$. Figure 1(b) shows a micrograph of the graphene flake before and after the four Ti/Au contacts were deposited in a cross pattern. The spacing of the contacts at the closest point was $1 \mu \mathrm{m}$. The carrier density was controlled by applying a voltage to the silicon back gate (a change of $1 \mathrm{~V}$ corresponds to a change in density of $7.1 \times 10^{10} \mathrm{~cm}^{-2}$ ). Standard low noise ac transport techniques were used to make the measurements. One of the four contacts briefly overlapped a bilayer. To eliminate any possibility that this influenced the results, the measurements were repeated with this particular contact used both as voltage and current contact. In each case qualitatively identical results were obtained. Figure 1(c) shows the Dirac curve obtained using the van der Pauw technique. Without any annealing the CNP occurred at $+0.75 \mathrm{~V}$. This small deviation suggests the absence of a significant incidental charged impurity local environment. To confirm the monolayer nature of the flake the quantum Hall effect was measured. This is shown in Fig. 1(d). The well-established graphene monolayer sequence $h /\left[(4 n+2) e^{2}\right]$ is observed. Magnetic field sweeps taken at high constant 1.96 $\times 10^{12} \mathrm{~cm}^{-2}$ electron density revealed $\mathrm{SdH}$ features down to the $h / 22 e^{2}$ (i.e., $n=5$ ) plateau. From these characterizations we estimate the carrier mobility to be $19000 \mathrm{~cm}^{2} / \mathrm{V}$ s close to the CNP and $5300 \mathrm{~cm}^{2} / \mathrm{V} \mathrm{s}\left(5150 \mathrm{~cm}^{2} / \mathrm{V} \mathrm{s}\right)$ at an electron (hole) density of $7.3 \times 10^{11} \mathrm{~cm}^{-2}$. We note, however, that no QHE feature was visible at any densities below $2 \mathrm{~T}$.

Conductance fluctuation measurements taken close to the CNP at low magnetic fields were visible with a root-meansquare exponential dependence up to $60 \mathrm{~K}$, as shown in Fig. 2 . The detailed pattern of fluctuations was altered on cycling the temperature between 4 and $100 \mathrm{~K}$. Such properties are consistent with a quantum interference origin for the fluctuations. We also observe a broad resistance dip in magnetic field sweeps close to the CNP (disappearing at a density of $10^{11} \mathrm{~cm}^{-2}$ ). This dip remained even at $100 \mathrm{~K}$ confirming that it was not related to a broad antiweak localization. Evidence for such an antiweak localization feature in addition to a weak localization peak was obtained by subtracting the lowtemperature curves from the $100 \mathrm{~K}$ data. A resistance dip which persisted to higher temperature has been previously reported in the literature and modeling found it to be consistent with the existence of electron and hole puddles. ${ }^{13}$ This is consistent with our data as we were able to obtain an excellent fit to our dip using Eq. (3) from Ref. 13 if we used 

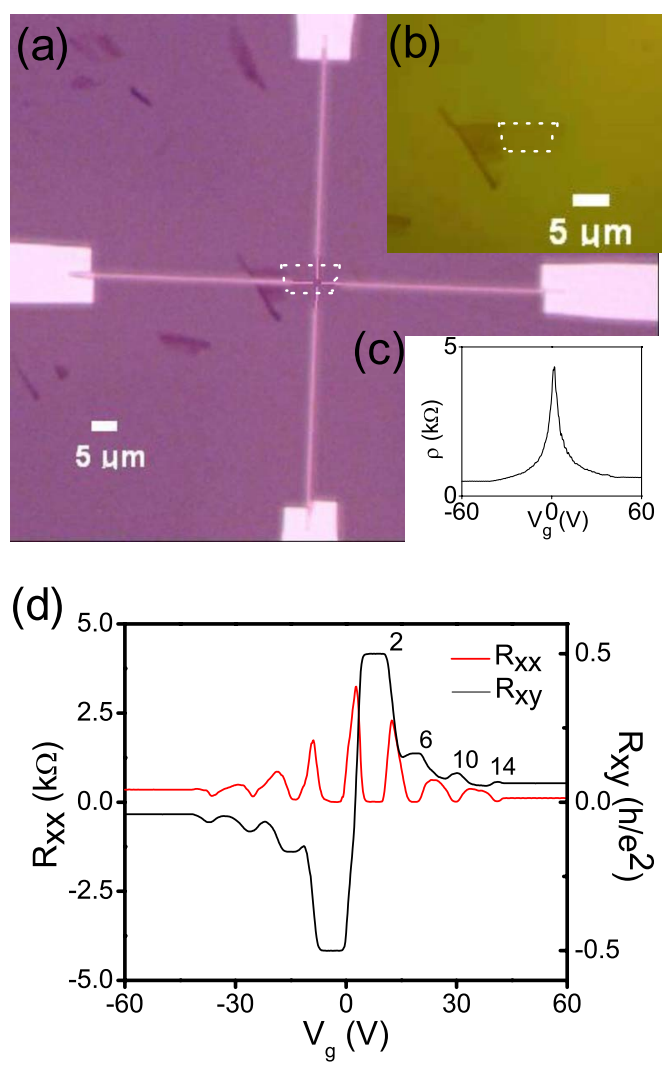

FIG. 1. (Color online) (a) Picture of the graphene flake with deposited leads; notice the fine leads making a cross pattern in the very center. The distance between leads at their closest point is on the order of $1 \mu \mathrm{m}$. (b) Optical image of graphene flake, with the monolayer region outlined in white. (c) Resistance against back gate voltage at $4.2 \mathrm{~K}$. (d) Longitudinal and transverse resistivities versus back gate voltage at $9 \mathrm{~T}$, showing quantum Hall plateaus at filling factors $\nu=4 n-2$.

slightly different parameters for each field direction (attributed to our contact geometry). The equation is based on an exact two-fluid model ${ }^{14}$ with an additional phenomenological conductivity term,

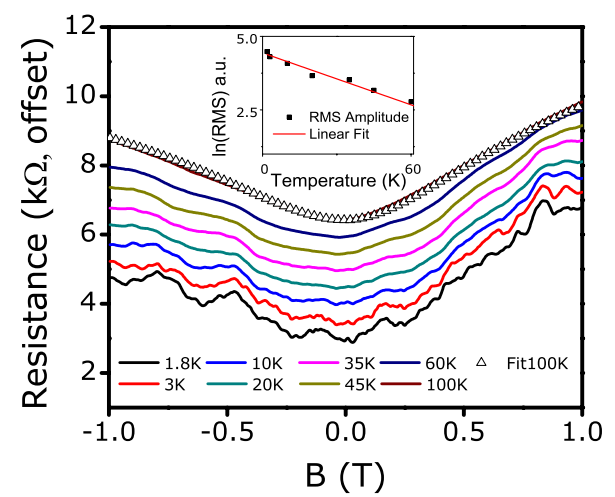

FIG. 2. (Color online) Longitudinal resistance as a function of magnetic field for different temperatures. Oscillations are present up to $60 \mathrm{~K}$. The $1.8 \mathrm{~K}$ curve is at its actual value with other curves offset by $500 \Omega$. Inset: linear fit to the logarithm of the rms amplitude of the oscillations, revealing their exponential characteristic versus temperature.
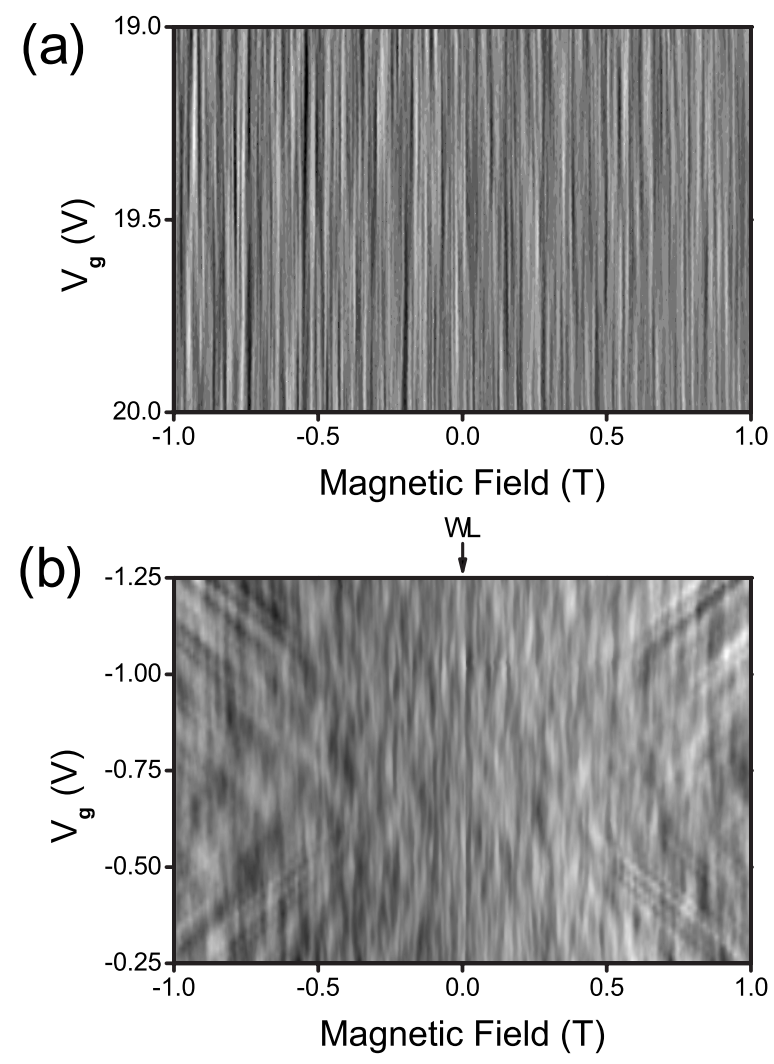

FIG. 3. (a) Numerical derivative versus magnetic field $d R_{x x} / d B$ for different density values in the electron regime. Black is minimal $d R_{x x} / d B$ while white is maximal $d R_{x x} / d B$. (b) Similar plot, at densities around the CNP. CNP is at $-0.75 \mathrm{~V}$.

$$
\rho_{x x}(B)=\left(\sigma_{x x, 1}+\frac{\sigma_{x x, 0}}{\left[1+(\mu B)^{2}\right]^{1 / 2}}\right)^{-1},
$$

with parameters for the left (right) side of the curve being $\sigma_{x x, 1}=2.68(1.90) e^{2} / h, \quad \sigma_{x x, 0}=6.17(6.95) e^{2} / h, \quad$ and $\mu$ $=2.63(2.93) \mathrm{m}^{2} / \mathrm{V} \mathrm{s}$. The fit over the $100 \mathrm{~K}$ trace is shown in Fig. 2. Although the equation is strictly only valid at the $\mathrm{CNP}$ we find phenomenologically that the density range of the dip $\left(2 \times 10^{11} \mathrm{~cm}^{-2}\right)$ is consistent with the range of the puddle regime (see below) as obtained from the $\mathrm{QH}$ dot charging events.

We first consider the behavior of the conductance fluctuations away from the CNP. Figure 3(a) shows the fluctuations from a density of $1.89 \times 10^{12}-1.96 \times 10^{12} \mathrm{~cm}^{-2}$ between -1 and $1 \mathrm{~T}$. The data are shown as a gray scale of its field derivative. Fast Fourier transforms (FFTs) performed in these data show no significant changes if the field range is increased from 0.5 to $1 \mathrm{~T}$. Figure $3(\mathrm{~b})$ is the equivalent plot but around the CNP. In this regime, the conductance fluctuations evolve dramatically over small changes in density. A series of parallel lines all pointing toward the CNP is observed in all four quadrants of the data at fields greater than $0.5 \mathrm{~T}$. The amplitude of the conductance fluctuations around the CNP is slightly higher than at higher carrier densities reaching $6 \%$ of the total conductance compared to $2 \%$ at $1.89 \times 10^{12} \mathrm{~cm}^{-2}$.

In Fig. 4(a), we plot the raw data minus a background, which removes the dip feature (N.B., the same background is 
(a)
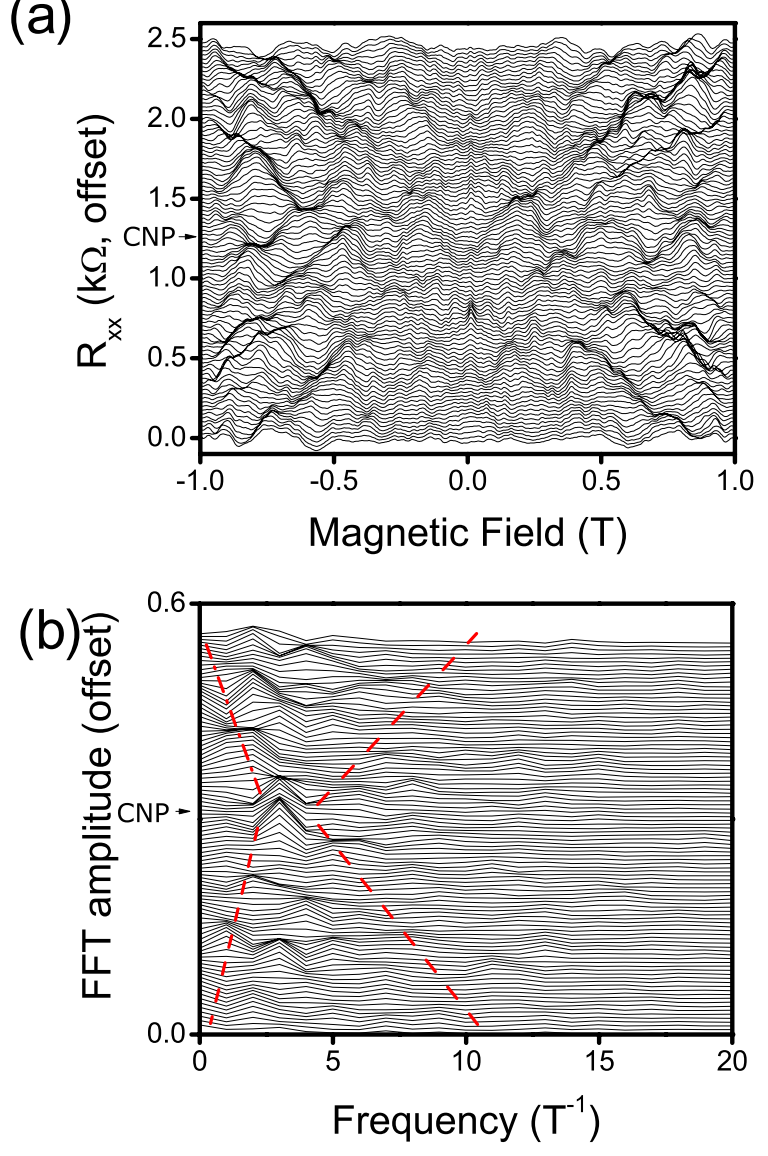

FIG. 4. (Color online) (a) Waterfall diagram of fluctuations in $R_{x x}$ with background removed around the CNP. (b) Fourier transforms of different lines in the waterfall diagram restricted to $\pm 0.5 \mathrm{~T}$ (see text or details). Red dashed lines are a guide to the eye emphasizing the narrowing of the fluctuation range at the CNP.

removed for all data). It is instructive to look at the FFTs as one moves through the CNP. The FFT plots, as seen Fig. 4(b) (where the data are restricted to $\pm 0.5 \mathrm{~T}$ to avoid the regions with diagonal lines), show that the spread in frequencies is narrowest at the CNP and increases in either direction as the gate voltage is tuned away from the CNP. Such a behavior is evidence that the fluctuations reflect the size of the puddles in the coexistence regime. At the CNP one might expect the sizes of electron and hole puddles to be relatively similar. As one moves away from the CNP toward more positive (negative) voltages the size of electron (hole) puddles grows (shrinks) leading to a wider spread of frequencies. Within this picture the dominant peak at the CNP in the FFT provides a measure of the spatial extent of the underlying potential fluctuations. In our measurements, this suggests a value of $125 \mathrm{~nm}$ for the puddle size. While our results are totally consistent with such a scenario, we caution that the underlying magnetic field period is only slightly bigger than a quarter of the field range. The size we calculate compares with estimates from 30 to $150 \mathrm{~nm}$ from scanning probe experiments. 5,15

To elucidate the nature of the parallel lines we plot, in Fig. 5 , the magnetoconductance over wider magnetic field and density ranges effectively expanding the measurements of

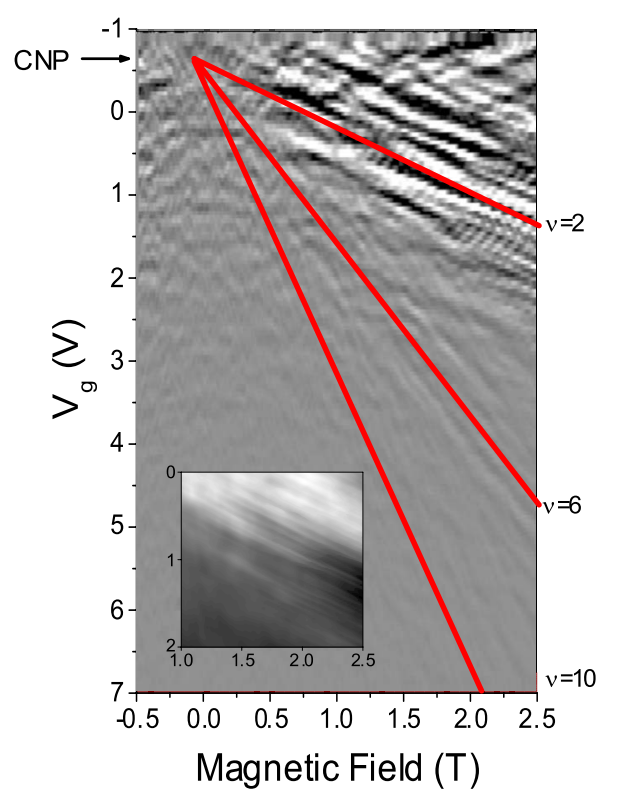

FIG. 5. (Color online) Gray scale of numerical derivatives $d^{2} R_{x x} / d B^{2}$, up to high electron densities. Red solid lines show the theoretical positions of filling factors of 2, 6, and 10 lines, from top to bottom. Inset shows a higher-resolution plot of the raw data for the upper right region.

the bottom right quadrant of Fig. 3(b). A careful examination of the magnetic field derivative of this plot reveals two additional much weaker sequences of parallel lines. Also shown on the plot are calculated lines corresponding to filling factors of 2, 6, and 10 of the quantum Hall effect. It is clear that the slopes of the three sets of parallel lines match up exactly with these filling factors. In the inset of Fig. 5, we show a higher-resolution resistance measurement (i.e., not the derivative) taken in one region of the plot. The full number of parallel features is more evident in these data.

We now address the origin of the lines corresponding to QHE filling factor slopes. To break down, the QHE requires edge states on opposite sides of the sample to equilibrate via backscattering events. In narrow samples this can occur resonantly via potential fluctuations. Cobden et al. ${ }^{16}$ showed in small metal-oxide-semiconductor field-effect transistors that at high magnetic fields such resonant backscattering events followed lines parallel to the $\mathrm{QH}$ filling factors. They proposed a model involving interactions. The potential inhomogeneity screening ability of the 2DEG is removed as the Landau level locally approaches full occupation. This leads to small quantum dots (QH quantum dots) isolated by incompressible regions. The backscattering events occur as the fluctuations are charged with single electrons in the same way that current flows through quantum dots at Coulomb blockade peaks. Such a charging picture has recently been confirmed by state-of-the-art probe experiments in AlGaAs/ GaAs and graphene 2DEGs. ${ }^{17,18}$ The probe experiments measured the local compressibility of these fluctuations directly. Our parallel lines originate at magnetic fields four times lower than any QHE feature and so a simple backscattering model associated with the breakdown of the QHE cannot be trivially evoked. We propose that the parallel lines 
are a precursor to the $\mathrm{QHE}$, which is possible due to the very wide density spread that occurs in graphene devices. From the spread in parallel lines we estimate density fluctuations of $2 \times 10^{11} \mathrm{~cm}^{-2}$ for electrons consistent with that obtained from local probe techniques. ${ }^{15,17}$ This results at low fields in a complex extended state network connecting closely separated contacts at low fields. Isolated $\mathrm{QH}$ dots can form "locally" in this regime. At these low magnetic fields charging of these dots does not backscatter current in the sense of the resonant breakdown of the quantum Hall effect. However, local equilibration redistributes the current in the current path network. These events are detected as fluctuations by the voltage probes. Amazingly the large density fluctuations in graphene, $2 \times 10^{11} \mathrm{~cm}^{-2}$, enable this type of conductance fluctuations to be observable for $2 \mathrm{~T}$ before even the $\mathrm{SdH}$ density of state oscillations is detected. Since sets of equally spread lines correspond to the charging of individual quantum dot states, we can estimate the size of the localized states. We find sets of up to five equally spaced lines. A typical quantum dot size we obtain in this analysis is about $160 \mathrm{~nm}$ (compared to $60 \mathrm{~nm}$ of Martin et al. ${ }^{17}$ using probe techniques). With a different contact arrangement (i.e., probing a different area of the graphene flake) we observed quali- tatively identical results but with a few fluctuations at twice the density spread of the results above, consistent with the mesoscopic nature of the fluctuations.

In conclusion, we have studied magnetoconductance fluctuations in a graphene monolayer close to the charge neutrality point. We find that the large density fluctuations in graphene lead to two separate categories for magnetoconductance fluctuations at low fields: those related to quantum interference and those related to charging of localized quantum Hall states. The spread in the quantum interference fluctuations in FFT plots narrows as one approaches the CNP suggesting that for these fluctuations it is the size of electron or hole puddles that is important rather then the overall density fluctuation range. During the course of this work, we became aware of similar results on graphene $p n p$ junctions by Velasco et al. ${ }^{19}$

We would like to acknowledge important motivating discussions with Louis Gaudreau, Ghislain Granger, Pawel Hawrylak, Devrim Guclu, Josh Folk, and Mark Lundeberg. A.S.S. and F.M.P. acknowledge funding from CIFAR. A.S.S. and S.B. acknowledge assistance from NSERC.
${ }^{1}$ K. S. Novoselov, A. K. Geim, S. V. Morozov, D. Jiang, Y. Zhang, S. V. Dubonos, I. V. Grigorieva, and A. A. Firsov, Science 306, 666 (2004).

${ }^{2}$ A. K. Geim and K. S. Novoselov, Nature Mater. 6, 183 (2007).

${ }^{3}$ A. H. Castro Neto, F. Guinea, N. M. R. Peres, K. S. Novoselov, and A. K. Geim, Rev. Mod. Phys. 81, 109 (2009).

${ }^{4}$ J. H. Chen, C. Jang, S. Adam, M. S. Fuhrer, E. D. Williams, and M. Ishigami, Nat. Phys. 4, 377 (2008).

${ }^{5}$ J. Martin, N. Akerman, G. Ulbricht, T. Lohmann, J. H. Smet, K. Von Klitzing, and A. Yacoby, Nat. Phys. 4, 144 (2008).

${ }^{6}$ V. Skakalova, A. B. Kaiser, J. S. Yoo, D. Obergfell, and S. Roth, Phys. Rev. B 80, 153404 (2009).

${ }^{7}$ S. V. Morozov, K. S. Novoselov, M. I. Katsnelson, F. Schedin, L. A. Ponomarenko, D. Jiang, and A. K. Geim, Phys. Rev. Lett. 97, 016801 (2006).

${ }^{8}$ C. Berger et al., Science 312, 1191 (2006).

${ }^{9}$ A. Rycerz, J. Tworzydlo, and C. W. J. Beenakker, EPL 79, 57003 (2007).

${ }^{10}$ D. W. Horsell, A. K. Savchenko, F. V. Tikhonenko, K.
Kechedzhi, I. V. Lerner, and V. I. Fal'ko, Solid State Commun. 149, 1041 (2009).

${ }^{11}$ F. V. Tikhonenko, D. W. Horsell, R. V. Gorbachev, and A. K. Savchenko, Phys. Rev. Lett. 100, 056802 (2008).

${ }^{12}$ E. McCann, K. Kechedzhi, V. I. Fal'ko, H. Suzuura, T. Ando, and B. L. Altshuler, Phys. Rev. Lett. 97, 146805 (2006).

${ }^{13}$ S. Cho and M. S. Fuhrer, Phys. Rev. B 77, 081402(R) (2008).

${ }^{14}$ V. Guttal and D. Stroud, Phys. Rev. B 71, 201304(R) (2005).

${ }^{15}$ J. Berezovsky, M. F. Borunda, E. J. Heller and R. M. Westervelt, arXiv:0907.0428 (unpublished).

${ }^{16}$ D. H. Cobden, C. H. W. Barnes, and C. J. B. Ford, Phys. Rev. Lett. 82, 4695 (1999).

${ }^{17}$ J. Martin, N. Akerman, G. Ulbricht, T. Lohmann, K. Von Klitzing, J. H. Smet, and A. Yacoby, Nat. Phys. 5, 669 (2009).

${ }^{18}$ S. Ilani, J. Martin, E. Teltelbaum, J. H. Smet, D. Mahalu, V. Umansky, and A. Yacoby, Nature (London) 427, 328 (2004).

${ }^{19}$ J. Velasco, G. Liu, L. Jing, P. Kratz, W. Bao, M. Bockrath, and C. Lau, Phys. Rev. B 81, 121407 (2010). 